\title{
Influence of genes and the environment in familial congenital heart defects
}

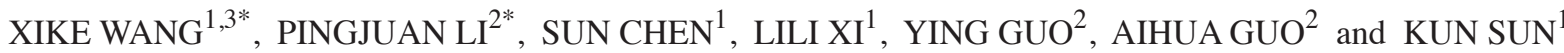 \\ ${ }^{1}$ Children's Heart Center, Xinhua Hospital Affiliated with Shanghai Jiao Tong University School of Medicine, \\ Shanghai 200092; ${ }^{2}$ Department of Cardiology, Shanghai Children's Medical Center \\ Affiliated with Shanghai Jiao Tong University School of Medicine, Shanghai 200127; \\ ${ }^{3}$ Department of Pediatrics, Guizhou Provincial People's Hospital, Guiyang, \\ Guizhou 550002, P.R. China
}

Received July 11, 2013; Accepted November 28, 2013

DOI: $10.3892 / \mathrm{mmr} .2013 .1847$

\begin{abstract}
The present study aimed to investigate genetic and environmental factors involved in the pathogenesis of congenital heart disease (CHD). A total of 61 familial pedigrees with CHD were analyzed, and 134 patients out of 761 family members had a diagnosis of CHD confirmed. The present study revealed that the prevalence of CHD in first-degree relatives $(55 / 249,22.0 \%)$ was significantly higher than that in second-degree relatives (18/526, 3.4\%). Additionally, the recurrence rate of CHD in families in which the patient's mother (12/61) or sister (15/61) had CHD were significantly higher than in cases with the father (6/61) or brother $(4 / 61)$ having CHD. The subtypes of CHD with increased risk of recurrence were ventricle septal defect (VSD) and atrial septal defect (ASD), followed by patent ductus arteriosus and tetralogy of fallot (TOF). In the 21 sets of twins among the 61 familial pedigrees analyzed, the concordance of both twins affected by CHD in identical and dizygotic twins was $94.4 \%(17 / 18)$ and $33.3 \%$ (1/3), respectively. Identical subtypes of CHD were identified in 10 out of 21 sets of twins. Of note, the following pattern was identified in three sets of the twins: One twin had TOF, while the other one had VSD. A risk factor survey revealed that threatened abortion in early pregnancy was associated with familial CHD. In conclusion, genetic factors may have important roles in the development of CHD, and TOF and VSD may have similar molecular mechanisms. Threatened abortion in early pregnancy is a novel environ-
\end{abstract}

Correspondence to: Dr Kun Sun, Children's Heart Center, Xinhua Hospital Affiliated with Shanghai Jiao Tong University School of Medicine, 1665 Kongjiang Road, Shanghai 200092, P.R. China

E-mail: sunkun@xinhuamed.com.cn

${ }^{*}$ Contributed equally

Key words: congenital heart disease, familial history, recurrence rate, risk factors, twins mental factor that may be specific in Chinese females with CHD.

\section{Introduction}

Congenital heart disease (CHD) is the most common congenital defect (1). It is also the most common cause of mortality associated with congenital defects in newborns and is often associated with fetal loss. The prevalence of CHD has increased from $0.6 \%$ in the 1930 s to $0.91 \%$ post- 1995 ; however, this may, in part, be a result of improved diagnosis and increased frequency of monitoring. The highest rate of CHD prevalence was reported to be 9.3 cases per 1,000 live births in Asia (2). Although certain minor abnormalities, such as a minor ventricle septal defect (VSD) or atrial septal defect (ASD), do not have any functional impact, rarely affect the quality of patients' lives and do not require intervention, the most complicated conditions require surgery or catheter intervention treatment. Certain complex lesions require multiple surgical procedures and close monitoring during the first few years of life. However, the etiology of CHD and its root cause remain to be elucidated. Therefore, exploration of genetic and environmental factors that may lead to an increased risk of CHD is of interest to patients with CHD and their families in terms of healthcare management and family planning. With the increasing number of patients with CHD, the assessment of the risk of recurrence in the children of patients with CHD has also become increasingly important (3). Furthermore, an enhanced understanding of CHD etiology may facilitate CHD prevention and clinical management of patients with CHD.

Approximately $20 \%$ of the cases of $\mathrm{CHD}$ result from known causes, such as genetic syndromes and teratogens. By contrast, little is known about the causes of the CHD in the remaining $80 \%$ of cases. It was hypothesized that these CHD lesions may be the result of interactions between genetic and environmental factors (4). Studies on CHD in the past decade have demonstrated that gene mutations of transcription factors, including T-box transcription factor 5 (TBX5), NK2 transcription factor related locus 5 protein (NKX2.5), and GATA binding protein 4 (GATA4), are able to cause CHD (5-8). 
A limited number of studies have examined the association between maternal lifestyle and risk of CHD. Maternal alcohol consumption during pregnancy is associated with birth defects in children (9-11). In the Baltimore-Washington Infant Study (12), maternal cigarette smoking of more than one pack per day was associated with two cardiac defects: Transposition with VSD and pulmonic stenosis (PS) (in females $>34$ years old) (12). Environmental factors affecting the rate of CHD have frequently been observed to act within the maternal preconceptional or fetal-placental-maternal context. The most common maternal risk factor is diabetes (13). Botto et al (14) reported that folate and folic acid-containing vitamin supplementation at the preconceptional stage has a protective effect against CHD.

The progress of novel genetic testing technologies, such as whole exome and genome sequencing, has greatly accelerated gene identification and led to the determination of novel candidate genes that may be involved in cases of CHD with unknown etiology (15). Traditional disease-gene linkage determination is predominantly based on analyzing a large number of families with multiple affected members, which is not practical for the analysis of CHD due to its rarity. A previous study conducted by our group analyzed 25 out of 61 familial pedigrees and found that the morbidity of CHD among the family members correlated with their genetic relationship, i.e. the risk of $\mathrm{CHD}$ was increased with the closeness of their genetic relationship. In addition, there was an increased incidence of threatened abortion in early pregnancy in patients with familial CHD as compared with those with sporadic CHD (16). Therefore, the present study design extended the familial pedigree collection and included additional families, as well as families with twins affected by $\mathrm{CHD}$, to further investigate the genetic relationships among affected patients, their family members with $\mathrm{CHD}$, familial CHD history and six key environmental factors. Results from the present study provide insights into the pathogenesis, intervention and prevention of CHD in China.

\section{Materials and methods}

Patients. The 61 familial CHD pedigrees, including 21 sets of twins, were diagnosed at the Shanghai Children's Medical Center (Shanghai, China) and Xinhua Hospital (Shanghai, China) between January 2009 and November 2012. Families containing at least two patients with CHD were defined as a family pedigree and included in this study. Detailed family history and risk factors were documented by trained staff using questionnaires. Parents and siblings of the probands with CHD were defined as first-degree relatives, while their grandparents, uncles, aunts and cousins were second-degree relatives. Informed written consent was obtained from each individual prior to data collection, and all investigational protocols were approved by the Human Ethics Committee (HEC) of the two Hospitals.

Methods. Surveys were conducted by trained medical staff to collect information on personal and family history of the patients with CHD. Comprehensive physical examination and echocardiography, chest X-ray, electrocardiography, cardiac catheterization or surgery were performed to further examine the first- and second-degree relatives of the probands previously diagnosed with CHD.

Family genetic information was obtained from the probands. Familial pedigree trees were constructed based on the information collected. A case-control study was conducted to identify the differences of environmental risk factors between familial and sporadic CHD patients. Sporadic control individuals were recruited in the hospitals at the same time, matched by gender, age and cardiac phenotype.

A case-control study was conducted to identify the differences in environmental risk factors between patients with familial and sporadic CHD. Six key environmental risk factors were included in the questionnaire: a) Moving into a new home or living in a new non-wood furniture home at any time between six months prior to pregnancy to the birth of the child; b) intense noise in the working and living environment during pregnancy; c) living in a room heated with charcoal or coal cinder, or with a high concentration of $\mathrm{CO}_{2}$ and $\mathrm{SO}_{2}$ for a prolonged time during pregnancy; d) smoking or passive smoking; e) drinking alcohol; f) suffering from respiratory infections during the first trimester of pregnancy; g) threatened abortion in early pregnancy. In total, 84 and 98 surveys were collected from patients with sporadic and familial CHD, respectively.

Statistical analysis. Comparisons were performed using Pearson's $\chi^{2}$ or Fisher's exact tests, and were based on two-tailed probability. $\mathrm{P}<0.05$ was considered to indicate a statistically significant difference. All analyses were performed using the SPSS 13.0 software package (SPSS, Inc., Chicago, IL, USA).

\section{Results}

Prevalence of $C H D$ in first- and second-degree relatives of probands. The incidence rates of CHD in all family members, first- and second-degree relatives of the probands were $9.4 \%(73 / 775), 22.0 \%(55 / 249)$ and $3.4 \%(18 / 526)$, respectively (Table I). The difference in the prevalence of CHD between the first- and second-degree relatives was statistically significant $(\mathrm{P}<0.01)$, and the difference between twins $(18 / 21,85.7 \%)$ and siblings (excluding twins) $(19 / 85,22.4 \%)$ was also significant $(\mathrm{P}<0.01)$.

Recurrence risk in patients with familial CHD. The prevalence of CHD in the fathers and mothers of the probands was $9.8 \%(6 / 61)$ and $19.7 \%(12 / 61)$, while the prevalence in brothers and sisters was $4.7 \%$ (4/85) and $17.6 \%$ (15/85), respectively. The difference in prevalence between brothers and sisters was statistically significant $(\mathrm{P}<0.01)$. The overall prevalence of $\mathrm{CHD}$ in first-degree male relatives of previously diagnosed patients with CHD $[6.8 \%$ (10/146)] was significantly lower than that in female first-degree relatives [18.5\% (27/146)]; $(\mathrm{P}<0.01)$ (Table II).

The recurrence rate of CHD also varied among different subtypes of CHD, with VSD and ASD being observed at the highest rates of recurrence $[24.6 \%(15 / 61)$ and $23.0 \%(14 / 61)$, respectively], followed by patent ductus arteriosus (PDA) and tetralogy of fallot (TOF) at 3.3\% (2/61). Aortic stenosis (AS), complete atrioventricular canal (CAVC), hypoplastic left heart syndrome (HLHS), interrupted aortic arch (IAA) and atrial septal defect/pulmonary atresia (ASD/PS) exhibited identical recurrence rates $(1.6 \%, 1 / 61)$ (Table III). 
Table I. Prevalence of CHD in first- and second-degree relatives of probands with CHD.

\begin{tabular}{lccc}
\hline Relation & Total number & Patients (n) & Prevalence $(\%)$ \\
\hline First-degree relatives & 249 & 55 & $22.0^{\mathrm{a}}$ \\
Parents & 122 & 18 & 14.6 \\
Twins & 42 & 18 & $42.9^{\mathrm{b}}$ \\
Siblings (excluding twins) & 85 & 19 & $22.4^{\mathrm{b}}$ \\
Second-degree relatives & 526 & 18 & $3.4^{\mathrm{a}}$ \\
Grandparents & 236 & 5 & 2.1 \\
Uncles/aunts & 196 & 4 & 2.0 \\
Cousins & 94 & 9 & 9.5 \\
Total & 775 & 73 & 9.4 \\
\hline
\end{tabular}

${ }^{\mathrm{a}} \chi^{2}=69.0, \mathrm{P}<0.01 ;{ }^{\mathrm{b}} \chi^{2}=29.8, \mathrm{P}<0.01 . \mathrm{CHD}$, congenital heart disease.

Table II. Gender distribution of first-degree relatives with CHD of probands with CHD.

A, Parents and siblings

\begin{tabular}{lccc}
\hline Group & Prevalence, $\%(\mathrm{n} /$ total $\mathrm{n})$ & $\chi^{2}$ & P-value \\
\hline Parents & $29.5(18 / 61)$ & $2.4^{\mathrm{c}}$ & $>0.05$ \\
Fathers & $9.8(6 / 61)$ & & \\
Mothers & $19.7(12 / 61)$ & & \\
Siblings & $22.3(19 / 85)$ & $7.2^{\mathrm{d}}$ & $<0.01$ \\
Brothers & $4.7(4 / 85)$ & & \\
Sisters & $17.6(15 / 85)$ & & \\
$\chi^{2 \mathrm{a}}$ & 1.0 & & \\
P-value & $>0.05$ & \\
\hline
\end{tabular}

$\mathrm{B}$, Males and females

\begin{tabular}{lccc}
\hline Group & Prevalence, $\%(\mathrm{n} /$ total $\mathrm{n})$ & $\chi^{2}$ & P-value \\
\hline Males & $6.8(10 / 146)$ & $8.5^{\mathrm{e}}$ & $<0.01$ \\
Fathers & $9.8(6 / 61)$ & & \\
Brothers & $4.7(4 / 85)$ & & \\
Females & $18.5(27 / 146)$ & $0.1^{\mathrm{f}}$ & \\
Mothers & $19.7(12 / 61)$ & & \\
Sisters & $17.6(15 / 85)$ & & \\
$\chi^{2 \mathrm{~b}}$ & 8.9 & \\
P-value $^{\mathrm{b}}$ & $<0.01$ & \\
\hline
\end{tabular}

aparents vs. siblings; ' males vs. females. CHD, congenital heart dis-

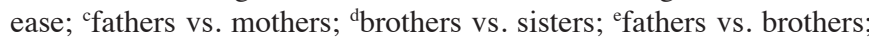
${ }^{\mathrm{f}}$ mothers vs. sisters.

CHD distribution and concordance in twins. In the present study, 21 sets of twins were included. In 18 sets, 17 of them monozygotic and one dizygotic, both twins were affected by CHD. By contrast, the case of only one twin from a set of
Table III. Recurrence of different CHD subtypes in 61 familial pedigrees.

CHD subtype

Recurrence, $\%$ (n/total $n)$

\begin{tabular}{lc}
\hline VSD & $24.6(15 / 61)$ \\
ASD & $23.0(14 / 61)$ \\
PDA & $3.3(2 / 61)$ \\
TOF & $3.3(2 / 61)$ \\
AS & $1.6(1 / 61)$ \\
CAVC & $1.6(1 / 61)$ \\
HLHS & $1.6(1 / 61)$ \\
IAA & $1.6(1 / 61)$ \\
ASD/PS & $1.6(1 / 61)$
\end{tabular}

VSD, ventricle septal defect; ASD, atrial septal defect; PDA, patent ductus arteriosus; TOF, tetralogy of fallot; AS, aortic stenosis; CAVC, complete atrioventricular canal; HLHS, hypoplastic left heart syndrome; IAA, interrupted aortic arch; PS, pulmonary atresia; CHD, congenital heart disease.

Table IV. CHD concordance in twins.

\begin{tabular}{lccc}
\hline Type & $\begin{array}{c}\text { Both } \\
\text { affected }\end{array}$ & $\begin{array}{c}\text { One } \\
\text { affected }\end{array}$ & $\begin{array}{c}\text { Concordance } \\
(\%)\end{array}$ \\
\hline Monozygotic twins & 17 & 1 & 94.4 \\
Dizygotic twins & 1 & 2 & 33.3 \\
\hline
\end{tabular}

CHD, congenital heart disease.

twins being affected by CHD was identified in only three out of the 21 sets of twins, two of them dizygotic and one monozygotic (Table IV).

Recurrent concordance of CHD (i.e., both twins had the same subtype of CHD) was identified in 10 sets of twins. Their CHD phenotypes were as follows: Four sets of twins had ASD; two sets of twins had VSD; one set of twins had HLHS; one had ASD/PS; one had TOF and one had IAA. 
Table V. Risk factor analysis in patients with sporadic and familial congenital heart disease.

\begin{tabular}{|c|c|c|c|c|c|c|}
\hline \multirow[b]{2}{*}{ Risk factors } & \multicolumn{2}{|c|}{ Sporadic } & \multicolumn{2}{|c|}{ Familial } & \multirow[b]{2}{*}{$\chi^{2}$} & \multirow[b]{2}{*}{ P-value } \\
\hline & Number (n) & Percentage $(\%)$ & Number (n) & Percentage (\%) & & \\
\hline \multicolumn{7}{|l|}{ a) New home } \\
\hline No & 77 & 91.7 & 93 & 97.9 & 0.77 & 0.38 \\
\hline Yes & 7 & 8.3 & 5 & 2.1 & & \\
\hline \multicolumn{7}{|l|}{ b) Noise } \\
\hline No & 77 & 91.7 & 96 & 98.0 & 2.59 & 0.11 \\
\hline Yes & 7 & 8.3 & 2 & 2.0 & & \\
\hline \multicolumn{7}{|c|}{ c) Charcoal burning } \\
\hline No & 82 & 97.6 & 98 & 100.0 & 0.68 & 0.21 \\
\hline Yes & 2 & 2.4 & 0 & 0.0 & & \\
\hline \multicolumn{7}{|l|}{ d) Smoking } \\
\hline No & 68 & 81.0 & 89 & 90.8 & 3.71 & 0.054 \\
\hline Yes & 16 & 19.0 & 9 & 9.2 & & \\
\hline \multicolumn{7}{|l|}{ e) Drinking } \\
\hline No & 79 & 94.0 & 95 & 96.9 & 0.34 & 0.56 \\
\hline Yes & 5 & 6.0 & 3 & 3.1 & & \\
\hline \multicolumn{7}{|c|}{ f) Respiratory infection } \\
\hline No & 52 & 61.8 & 71 & 72.4 & 2.30 & 0.13 \\
\hline Yes & 32 & 38.2 & 27 & 27.6 & & \\
\hline \multicolumn{7}{|c|}{ g) Threatened abortion } \\
\hline No & 78 & 92.9 & 64 & 75.5 & 20.01 & $<0.001$ \\
\hline Yes & 6 & 7.1 & 34 & 24.5 & & \\
\hline
\end{tabular}

a, Moving into a new home or living in a new non-wood furniture home at any time between the six months prior to pregnancy to the birth of the child; b, intense noise in working and living environment during pregnancy; c, living in a room heated with charcoal or coal cinder, or with a high concentration of $\mathrm{CO}_{2}$ and $\mathrm{SO}_{2}$ for a prolonged time during pregnancy; d, smoking or passive smoking; e, drinking alcohol; f, suffering from respiratory infections during the first trimester of pregnancy; g, threatened abortion in early pregnancy.

Of note, three sets of twins had the same recurrent pattern: The probands had TOF and their identical twins had VSD. The phenotypes of three sets of twins in which only one of them was affected by CHD were as follows: a) One patient was affected by PS/right ventricular outflow tract obstruction (RVOTO)/VSD and his monozygotic brother was healthy; b) one was diagnosed with ASD/moderate regurgitation of the mitral valve/porosity of myocardial tissue, while his dizygotic sister was unaffected and c) one was affected by a double outlet of right ventricle and his dizygotic sister was unaffected. In addition, four sets of twins had different subtypes of CHD: One twin had PDA/patent foramen ovule (PFO) while the other one had PDA/PFO/pulmonary hypertension $(\mathrm{PH})$; one had ASD/PH/Down syndrome while her dizygotic brother had ASD, which spontaneously healed; one had VSD/PDA/PH while the other one had VSD; and one had PA/VSD/PS while the other one had VSD/PDA/PH.

Environmental risk factors. A total of 98 risk factor questionnaires were collected from patients with familial CHD and 84 from patients with sporadic CHD. The results showed that threatened abortion was significantly higher in the familial CHD group than in the sporadic group $(\mathrm{P}<0.05)$. Data were analyzed by the $\chi^{2}$ test; no significant differences were found among the remaining four risk factors (Table V).

\section{Discussion}

It is generally accepted that CHD is a multifactorial disease and that genetic and environmental factors are involved in the development of the disease (4). Genetic variations interact with each other, and, together with environmental factors, increase the susceptibility to CHD. A number of studies have focused on the etiology of CHD during the past decades $(17,18)$. However, the exact pathogenesis of $\mathrm{CHD}$ remains to be elucidated. Previous studies have revealed that CHD has the potential to aggregate in families with its recurrent risk in relatives being higher than in the general population and positively correlated with genetic relationship $(17,19)$. The results of the present study are in accordance with previous studies in terms of CHD being more likely to occur when the first-degree relatives of patients with CHD were affected with CHD. The CHD recurrence is significantly higher in the first-degree relatives compared with that of the second-degree relatives, implying that genetic factors have a key role in the development of CHD. 
A previous study reported that the risk of recurrence in children of families in which one or both of the parents are affected by CHD is significantly higher than in children whose siblings are affected and their parents unaffected by CHD. Furthermore, the risk is higher when the mother is affected by CHD, compared with when the father is affected (20). When both parents are unaffected by CHD, the recurrence risk in the siblings of an affected individual is $1-6 \%$ for the majority of CHD lesions $(21,22)$. The present study revealed that the recurrence risk of CHD was significantly higher in patients whose mother rather than father was affected, which suggested that maternal genetic factors may contribute to the development of CHD. By contrast, no difference in the risk of recurrence between individuals whose parents were affected and those whose siblings were affected was identified. These findings are rather inconclusive due to the limited number of patients and family members in the present study. However, to the best of our knowledge, the present study was the first to show that the recurrence risk was higher in patients whose sister was affected than in those whose brother was affected $(\mathrm{P}<0.01)$. This is another indication of the influence of maternal genetic factors in the development of CHD.

The twin studies conducted in the present study, although limited by complicated factors, provided the most reliable source for separating genetic contributions to CHD from environmental influences. Monozygous (identical) twins have the same genetic background; however, they are raised separately under the influence of different environmental factors. The concordance rate in monozygotic twins was compared with that of dizygotic (fraternal) twins to evaluate the genetic component (heritability) of the development of CHD. In the 21 twins in the present study, the concordance rate in monozygotic twins was $94.4 \%$, while the concordance rate in dizygotic twins was only $33.3 \%$. These results emphasize the involvement of multiple factors in the development of CHD, despite the low group size of dizygotic twins $(n=3)$. It is of note that TOF and VSD occurred concurrently in three sets of twins, indicating that TOF and VSD may have similar mechanisms $(20,22)$.

Environmental factors associated with CHDs have been observed to act in a maternal preconceptional or fetal-placental-maternal context. As aforementioned, mothers with pre-gestational diabetes have a five-fold increased risk in giving birth to children with $\mathrm{CHD}$ (5). Other environmental factors, although not conclusive, have also been reported (9-11). Shillingford and Weiner (23) reported that ASD was the most frequent cardiac anomaly in neonates with fetal alcohol syndrome caused by maternal alcohol consumption. A case-control study in Spain showed that a higher risk of developing CHDs was present in a group with the highest level of prenatal exposure to alcohol (24). Furthermore, Torfs and Christianson (25) reported that maternal cigarette smoking was associated with specific defects (atrioventricular canal, ASD and TOF). However, another study found no association between any heart defect and maternal smoking (26). Therefore, the present study considered six major environmental factors relevant for investigating their association with the development of CHD. It was revealed that threatened abortion in early pregnancy was significantly higher in patients with familial CHD than in patients with sporadic CHD, while for smoking, we observed that was it marginally sigificantly assocaited with familial CHD ( $\mathrm{P}=0.05)$, which we consider to be due to the small sample size in the present study and therefore requires further study to investigate this association.

The present study confirmed previous findings and revealed additional mechanisms of CHD and potential environmental risk factors. Larger patient groups and a larger database of environmental factors are required to further verify the results of the present study. We aim to continue and extend the collection of pedigrees with detailed family histories, including the medical history of the mother and vitamin supplement intake during pregnancy. A total of 61 families have been enrolled in this continuing study and their blood samples have been collected for further analysis. Genetic analysis via next-generation sequencing and characterization of patients with CHD is ongoing.

In conclusion, the present study provided supportive information towards an enhanced understanding of the mechanism of the development of CHD. The results have laid a foundation to further investigate CHD-associated gene identification and provide a fundamental approach to establish individual genetic counseling for patients with CHD and their families. The presented analysis of pedigrees may facilitate research and clinical investigation to further explore and identify the pathogenic linkage of CHDs as well as the molecular mechanisms and genes involved in the development of CHD.

\section{Acknowledgements}

The authors gratefully acknowledge all the patients and their families who participated in the present study, and all the staff of the Heart Medical Center and Xinhua Hospital (Shanghai, China) for their assistance in sample collection. This study was supported by the National Basic Research Program of China (grant no. 2010CB529500), the Science and Technology Commission of Shanghai Municipality (grant no. 10DZ1951203), the program for the Innovative Research Team of Shanghai Municipal Education Commission (Phase I): Pediatrics, and the Key Discipline of Shanghai Municipal Education Commission Major (Phase III): Pediatric Cardiology.

\section{References}

1. Hoffman JI and Kaplan S: The incidence of congenital heart disease. J Am Coll Cardiol 39: 1890-1900, 2002.

2. van der Linde D, Konings EE, Slager MA, Witsenburg M, Helbing WA, Takkenberg JJ and Roos-Hesselink JW: Birth prevalence of congenital heart disease worldwide: a systematic review and meta-analysis. J Am Coll Cardiol 58: 2241-2247, 2011.

3. Fesslova V, Brankovic J, Lalatta F, Villa L, Meli V, Piazza L and Ricci C: Recurrence of congenital heart disease in cases with familial risk screened prenatally by echocardiography. J Pregnancy 2011: 368067, 2011.

4. Nora JJ: Multifactorial inheritance hypothesis for the etiology of congenital heart disease: the genetic-environmental interaction. Circulation 38: 604-617, 1968.

5. Basson CT, Bachinsky DR, Lin RC, et al: Mutations in human TBX5 [corrected] cause limb and cardiac malformation in Holt-Oram syndrome. Nat Genet 15: 30-35, 1997.

6. Schott JJ, Benson DW, Basson CT, Pease W, Silberbach GM, Moak JP, Maron BJ, Seidman CE and Seidman JG: Congenital heart disease caused by mutations in the transcription factor NKX2-5. Science 281: 108-111, 1998.

7. Garg V, Kathiriya IS, Barnes R, et al: GATA4 mutations cause congenital heart defects and reveal an interaction with TBX5. Nature 424: 443-447, 2003. 
8. Butler TL, Esposito G, Blue GM, et al: GATA4 mutations in 357 unrelated patients with congenital heart malformation. Genet Test Mol Biomarkers 14: 797-802, 2010.

9. Brent RL: Environmental causes of human congenital malformations: the pediatrician's role in dealing with these complex clinical problems caused by a multiplicity of environmental and genetic factors. Pediatrics 113 (4 Suppl): 957-968, 2004

10. Polifka JE and Friedman JM: Medical genetics: 1. Clinical teratology in the age of genomics. CMAJ 167: 265-273, 2002

11. Autti-Rämö I, Fagerlund A, Ervalahti N, Loimu L, Korkman M and Hoyme HE: Fetal alcohol spectrum disorders in Finland: clinical delineation of 77 older children and adolescents. Am J Med Genet A 140: 137-143, 2006.

12. Ferencz C, Loffredo CA, Correa-Villasenor A and Wilson PD (eds): Genetic and Environmental Risk Factors of Major Congenital Heart Disease: The Baltimore-Washington Infant Study 1981-1989. Vol 5. Futura Publishing Company, Armonk, NY, 1997.

13. Wren C, Birrell G and Hawthorne G: Cardiovascular malformations in infants of diabetic mothers. Heart 89: 1217-1220, 2003.

14. Botto LD, Olney RS and Erickson JD: Vitamin supplements and the risk for congenital anomalies other than neural tube defects. Am J Med Genet C Semin Med Genet 125C: 12-21, 2004.

15. Wolf M and Basson CT: The molecular genetics of congenital heart disease: a review of recent developments. Curr Opin Cardiol 25: 192-197, 2010.

16. Wang X, Wang J, Zhao P, Guo Y, Wu L, Sun J and Sun K: Familial congenital heart disease: data collection and preliminary analysis. Cardiol Young 23: 394-399, 2013
17. Hoess K, Goldmuntz E and Pyeritz RE: Genetic counseling for congenital heart disease: new approaches for a new decade. Curr Cardiol Rep 4: 68-75, 2002.

18. Bruneau BG: The developmental genetics of congenital heart disease. Nature 451: 943-948, 2008.

19. Øyen N, Poulsen G, Boyd HA, Wohlfahrt J, Jensen PK and Melbye M: Recurrence of congenital heart defects in families. Circulation 120: 295-301, 2009.

20. Burn J, Brennan P, Little J, et al: Recurrence risks in offspring of adults with major heart defects: results from first cohort of British collaborative study. Lancet 351: 311-316, 1998.

21. Calcagni G, Digilio MC, Sarkozy A, Dallapiccola B and Marino B: Familial recurrence of congenital heart disease: an overview and review of the literature. Eur J Pediatr 166: 111-116, 2007.

22. Corone P, Bonaiti C, Feingold J, Fromont S and Berthet-Bondet D: Familial congenital heart disease: how are the various types related? Am J Cardio 51: 942-945, 1983.

23. Shillingford AJ and Weiner S: Maternal issues affecting the fetus. Clin Perinatol 28: 31-70, 2001.

24. Martínez-Frías ML, Bermejo E, Rodríguez-Pinilla E and Frías JL: Risk for congenital anomalies associated with different sporadic and daily doses of alcohol consumption during pregnancy: a case-control study. Birth Defects Res A Clin Mol Teratol 70: 194-200, 2004.

25. Torfs CP and Christianson RE: Maternal risk factors and major associated defects in infants with Down syndrome. Epidemiology 10: 264-270, 1999.

26. Källén K: Maternal smoking and congenital heart defects. Eur J Epidomiol 15: 731-737, 1999. 